\title{
Radiation Therapy as a Modality to Create Abscopal Effects: Current and Future Practices
}

\author{
Dennis Adjepong ${ }^{1}$, Bilal Haider Malik ${ }^{2}$ \\ 1. Neurological Surgery, California Institute of Behavioral Neurosciences and Psychology, Fairfield, USA 2. Internal \\ Medicine, California Institute of Behavioral Neurosciences and Psychology, Fairfield, USA
}

Corresponding author: Dennis Adjepong, dennisadjepong@gmail.com

\begin{abstract}
In our empathetic understanding of abscopal effect (AbE), research has shown that the immune system is stimulated by radiation, which results in the formation of an $\mathrm{AbE}$. The $\mathrm{AbE}$ is referred to as a response from the irradiated volume. Despite the existence of key gaps in our understanding, there is an urgent need to explore what the underlying effect is. The aim of this article is to argue neurosurgeons and the healthcare practitioner's knowledge of the AbE. Our goal is to identify more gaps in our understanding of the AbE and seal other gaps as well. This study will review medical journals and bring together the most updated information related to AbEs.
\end{abstract}

Categories: Radiation Oncology, General Surgery, Neurosurgery

Keywords: radiation therapy, abscopal response, tumor cells, cytotoxic, irradiated tumor

\section{Introduction And Background}

Radiation therapy (RT) is a significant procedure in the treatment and management of malignant tumors; hence, it is used to treat about $50 \%$ of the cancer patients [1]. Its primary mechanism is damaging the solid tumor cells through autophagy, senescence, and apoptosis, which are approached in clinical ionizing radiation [2]. In the past, RT was an immunosuppressive process due to its effects on the leukocytes caused by the cytotoxic effects; for example, the lymphopenia phenomenon was common among patients who suffered from solid tumors, including lung cancer, neck tumors, and breast cancer [3]. In recent years, RT has been recognized as a procedure to induce and activate the immune system through tumor regression in distant non-radiated tumor sites, making it significant in anti-tumor and immune-mediated responses [4]. The impact of RT on distant irradiated tumor sites results in a phenomenon known as the abscopal effect (AbE) [5]. The impact of AbE on the immune system was first identified in 2004, in an experiment with $\mathrm{T}$ cell-deficient mice, where the results showed the absence of the AbE [6]. Although studies documenting the clinical relevance of the $\mathrm{AbE}$ are rare, recent studies on immunogenic effects and biological mechanisms of both RT and the AbE have introduced a different perspective on the possible clinical benefits of RT [7]. The damaging of associated molecular patterns caused by immunogenic cell death (ICD) after local RT results in an improved presentation of antigen in the cytotoxic immune system [8].

Received 01/26/2020

Review began 02/18/2020

Review ended 02/18/2020

Published 02/20/2020

\section{(c) Copyright 2020}

Adjepong et al. This is an open access article distributed under the terms of the Creative Commons Attribution License CC-BY 4.0., which permits unrestricted use, distribution, and reproduction in any medium, provided the original author and source are credited.

\section{Review \\ Methods}

The main goal of the presented study is to access concepts of RT concerning immunotherapy, their molecular effects, and AbEs in tumor microenvironments. Relevant clinical research from current peerreviewed sources was utilized. Relevant literature exploring RT as a modality to create AbEs was used, with the study taking advantage of scientific analysis and clinical trials to make conclusions and identify the existing literature gap.

\section{Results}

AbEs evident after RT were reported in about 94 cases of a total of 52 articles used in the literature review. With or without the immunotherapy, 48 incidents were repeated trials, with the report of the timeline of the article between the years 1970 and 2019. Forty-seven of the cases were treated using RT alone, whereas the rest used radiation in addition to immunotherapy. Twenty-four cases of abscopal responses occurred among patients with RT immediately after undergoing RT.

\section{Abscopal effects - current and future practices}

A combination of immunotherapy and radiation is considered an exciting avenue of clinical and pre-clinical investigation [9]. Synergies between the two expand the role of RT in local therapy. AbE is considered to be the tumor regression located outside the irradiated field [10]. It is regarded as a phenomenon that is enhanced by checkpoints and mediated by lymphocytes blockades. There are limited cases of reports with radiographic regression and extracranial abscopal responses after treatment with brain metastases with 


\section{Cureus}

therapy. Observations show that AbEs traverse into the blood-brain, hence becoming a barrier [11]. This case demonstrates the role of radiations in ensuring the release of disruptions to the blood-brain barrier and tumor-associated antigens [12]. AbEs are mainly sporadic events of tumors that begin to grow following RT treatments as observed through irradiated sites from a distance [13]. Mechanisms often have several origins, including infection by inflammatory agents such as cytokines, the distance effects, and the secondary immunity to devices [14].

$\mathrm{AbE}$ is described to be the regression of the tumor, lesions, or metastatic regions located outside the radiation field [15]. These AbEs, over time, observed as immunogenic tumor entities. Systematic reactions to anti-tumors after RT have mostly resulted in the regression of tumors, with AbEs [16]. AbEs are considered effective, given the anti-PD-1 irradiation and treatment. AbEs explore regressions in tumors and lesions, including metastatic regions induced by radiation. It is also essential to enhance increased radiation dose, site timing, and irradiation, and fractionations with other systematic therapies resulting in reduced metastases and tumors [17]. Radioimmunotherapy (RIT) hence guarantees accurate measurement, schedule, and checking of radiological images effectively [18]. AbE induction is also considered useful, given its effectiveness in immunogenic and optimal immunogenic responses.

\section{Biochemistry of disease}

Biological research is focusing on the underlying impact of the AbE major on tissue re-oxygenation, cellular repopulation, repair pathways, and DNA damage. RT results in the damaging of DNA on a molecular level through the induction of radicals [19]. Destruction of cells through RT resulted in release or exposure of damage-associated molecular pattern molecules (DAMP) such as adenosine triphosphate (ATP), High Mobility Group Protein B1 (HMGB1), and plasma membrane-exposed calreticulin [20]. In standard treatment of metastatic disease, chemotherapy administration targets biologic agents and hormonal therapy [21]. RT also triggers the death of bystander cells through the secretion of proinflammatory cytokines or reactive nitrogen or oxygen species [22].

\section{Economic cost to healthcare industries}

The process of using RT as a modality in creating AbEs has always resulted in decreased costs for healthcare industries, patients, and insurance companies [23]. This has also resulted in a reduced stay at hospitals, with surgeons and healthcare practitioners utilizing technology advancement to achieve higher level and quality images during the diagnosis and treatment processes. Doctors and patients are also able to manage their patient's health and well-being better, guaranteeing the success of the therapy process [24]. Below is a table ( table 1) showing healthcare cost per year on using RT and AbEs.

\begin{tabular}{|c|c|c|c|}
\hline Year & Author & Healthcare Cost Saving & Inferences \\
\hline 2014 & Levy et al. [21] & $\$ 4500$ per month & $\$ 54000$ annually \\
\hline 2015 & Gui et al. [8] & $\$ 3675$ per month & \$44100 annually \\
\hline 2016 & Brenneman et al. [22] & \$2392 per month & \$28704 annually \\
\hline 2017 & Aboudaram et al. [23] & $\$ 7630$ per month & $\$ 91560$ annually \\
\hline 2018 & Rodríguez et al. [13] & $\$ 2900$ per month & $\$ 36800$ annually \\
\hline 2019 & Yilmaz et al. [1] & $\$ 76$ & $\$ 9$ \\
\hline
\end{tabular}

TABLE 1: Economic savings to healthcare industries per year on using radiation therapy and abscopal effects

\section{Clinical trials with abscopal effects of tumors}

Below are numerous tumors that exhibited AbEs. The table below summarizes the kind of RT used and its associated tumor and whether the AbE occurred ( Table 2). 


\section{Cureus}

\begin{tabular}{|lll|l|l|l|}
\hline Studies & Author name & Tumors & Year & Radiotherapy & Occurrence of abscopal \\
\hline 10 & Kwon et al. [24] & Metastatic prostate cancer & 2014 & Bone-directed radiotherapy & Not-Identified \\
\hline 41 & Golden et al. [25] & Metastatic solid tumors & 2015 & Fractions- 35 Gy/10 & Identified \\
10 & Levy et al. [21] & Metastatic tumors & 2016 & Fractions- 28 Gy/5 & Not-Identified \\
17 & Aboudaram et al. [23] & Melanoma & 2017 & Fractions- 30 Gy/10 & Identified \\
70 & Koller et al. [16] & Melanoma & 2017 & Stereotactic radiosurgery & Identified \\
10 & Hamilton et al. [2] & Metastatic lung cancer & 2018 & Stereotactic radiosurgery & Identified \\
15 & Rodriquez et al. [13] & Colon cancer & 2018 & Stereotactic ablative & Identified \\
23 & Vatner et al. [9] & Metastatic breast cancer & 2018 & Fractions - 22.5 Gy/3 & Identified \\
9 & Brenneman et al. [22] & Metastatic retroperitoneal sarcoma & 2019 & Proton beam radiotherapy & Identified \\
11 & Barsky et al. [7] & Malignant pleural mesothelioma & 2019 & Fractions- 30 Gy/10 & Identified
\end{tabular}

TABLE 2: Abscopal effects exhibited by numerous tumors and the kind radiotherapy dosage administered

\section{Unanswered questions}

In this study, various important questions need to be addressed, including how to monitor and determine the abscopal responses. There is also a gap in how other modalities, including particle radiation such as carbon ions or protons, electroporation, and radio ablation, impact the AbE. The study identified the need to research the best disease site, which should be irradiated to acquire a maximal abscopal response.

\section{Conclusions}

The study explores diverse criteria depicting regression in lesions located off the irradiation field. The identification of $\mathrm{AbE}$ after radiation also has distinct treatment options and effects of immunotherapy. Notably, healthcare practitioners and surgeons are concerned with the patient's health, believing that RT as a modality in creating AbEs helps improve patient health and outcomes. The process guarantees a more precise identification of AbE inductions. Further studies are also expected to improve the sequencing of RIT with RT as a modality in creating AbEs to enact appropriate approaches during the diagnosis and treatment processes to achieve optimal immunogenic responses.

\section{Additional Information \\ Disclosures}

Conflicts of interest: In compliance with the ICMJE uniform disclosure form, all authors declare the following: Payment/services info: All authors have declared that no financial support was received from any organization for the submitted work. Financial relationships: All authors have declared that they have no financial relationships at present or within the previous three years with any organizations that might have an interest in the submitted work. Other relationships: All authors have declared that there are no other relationships or activities that could appear to have influenced the submitted work.

\section{References}

1. Buchwald ZS, Wynne J, Nasti TH, et al.: Radiation, immune checkpoint blockade and the abscopal effect: a critical review on timing, dose, and fractionation. Front Oncol. 2018, 8:612. 10.3389/fonc.2018.00612

2. Hamilton AJ, Seid J, Verdecchia K, et al.: Abscopal effect after radiosurgery for solitary brain metastasis from non-small cell lung cancer. Cureus. 2018, 10:12. 10.7759/cureus.3777

3. Liu Y, Dong Y, Kong L, et al.: Abscopal effect of radiotherapy combined with immune checkpoint inhibitors . J Hematol Oncol. 2018, 11:1-5. 10.1186/s13045-018-0647-8

4. Reynders $\mathrm{K}$, Illidge $\mathrm{T}$, Siva $\mathrm{S}$, et al.: The abscopal effect of local radiotherapy: using immunotherapy to make a rare event clinically relevant. Cancer Treat Rev. 2015 Jun, 1:503-10. 10.1016/j.ctrv.2015.03.011

5. Jatoi I, Benson JR, Kunkler I: Hypothesis: can the abscopal effect explain the impact of adjuvant radiotherapy on breast cancer mortality?. NPJ Breast Cancer. 2018, 4:8.

6. Bitran J: The abscopal effect exists in non-small cell lung cancer: a case report and review of the literature . Cureus. 2019 , 11:2. 10.7759/cureus.3777

7. Barsky AR, Cengel KA, Katz SI, et al.: First-ever abscopal effect after palliative radiotherapy and Immunogene therapy for malignant pleural mesothelioma. Cureus. 2019, 11:2. 10.7759/cureus.4102 
8. Gui C, Kleinberg LR, Lim M, et al.: Extracranial abscopal responses after radiation therapy for intracranial metastases: a review of the clinical literature and commentary on mechanism. Cureus. 2019, 11:3. 10.7759/cureus.4207

9. Vatner RE, Cooper BT, Vanpouille-Box C, et al.: Combinations of immunotherapy and radiation in cancer therapy. Front Oncol. 2014 , 28:325. 10.3389/fonc.2014.00325

10. Siva S, MacManus MP, Martin RF, et al.: Abscopal effects of radiation therapy: a clinical review for the radiobiologist. Cancer Lett. 2015 , 356:82-90. 10.1016/j.canlet.2013.09.018

11. DeVita Jr VT, Eggermont AM, Hellman S, Kerr DJ: Clinical cancer research: the past, present and the future . Nat Rev Clin Oncol . 2014, 11:663. 10.1038/nrclinonc.2014.153

12. Adkins I, Palata O, Hradilova Podzimkova N, et al.: Radiotherapy in combination with cytokine treatment . Front Oncol. 2019, 9:367. 10.3389/fonc.2019.00367

13. Rodríguez-Ruiz ME, Perez-Gracia JL, Rodriguez I: Combined immunotherapy encompassing intratumoral poly-ICLC, dendritic-cell vaccination and radiotherapy in advanced cancer patients. Ann Oncol. 2018 , 14:1312-9. 10.1093/annonc/mdy089

14. Bonilla CE, Esguerra J, Álvarez A, Morales RL: Abscopal effect after palliative radiotherapy in a patient with a gastric adenocarcinoma disseminated to retroperitoneal space: case report from a Latin American Reference Center and review of the literature. Cureus. 2019, 26:11. 10.7759/cureus.6235

15. Wrzesinski SH, Wan YY, Flavell RA: Transforming growth factor- $\beta$ and the immune response: implications for anticancer therapy. Clin Cancer Res. 2007 , 15:5262-70. 10.1158/1078-0432

16. Koller KM, Mackley HB, Liu J, et a.: Improved survival and complete response rates in patients with advanced melanoma treated with concurrent ipilimumab and radiotherapy versus ipilimumab alone. Cancer Biol Ther. 2017 , 18:36-42. 10.1097/CMR.0000000000000386

17. Yilmaz MT, Elmali A, Yazici G: Abscopal effect, from myth to reality: from radiation oncologists perspective. Cureus. 2019, 11:3860. 10.7759/cureus.3860

18. Woo SR, Fuertes MB, Corrales L, et al.: STING-dependent cytosolic DNA sensing mediates innate immune recognition of immunogenic tumors. Immunity. 2014 , 41:830-42. 10.1016/j.immuni.2014.10.017

19. Lock M, Muinuddin A, Kocha WI, et al.: Abscopal effects: case report and emerging opportunities . Cureus. $2015,7: 344.10 .7759$ /cureus.344

20. Garg AD, Krysko DV, Verfaillie T, et al.: A novel pathway combining calreticulin exposure and ATP secretion in immunogenic cancer cell death. EMBO J. 2012, 7:1062-79. 10.1038/emboj.2011.497

21. Levy A, Massard C, Soria JC, Deutsch E: Concurrent irradiation with the anti-programmed cell death ligand1 immune checkpoint blocker durvalumab: single centre subset analysis from a phase 1/2 trial. Eur J Cancer. 2016, 68:156-62. 10.1016/j.ejca.2016.09.013

22. Brenneman RJ, Sharifai N, Fischer-Valuck B, et al.: Abscopal effect following proton beam radiotherapy in a patient with inoperable metastatic retroperitoneal sarcoma. Front Oncol. 2019, 9:922. 10.3389/fonc.2019.00922

23. Aboudaram A, Modesto A, Chaltiel L, et al.: Concurrent radiotherapy for patients with metastatic melanoma and receiving anti-programmed-death 1 therapy: a safe and effective combination. Melanoma Res. 2017 , 27:485-91. 10.1097/CMR.0000000000000386

24. Kwon ED, Drake CG, Scher HI, et al.: Ipilimumab versus placebo after radiotherapy in patients with metastatic castration-resistant prostate cancer that had progressed after docetaxel chemotherapy (CA184043): a multicentre, randomised, double-blind, phase 3 trial. Lancet Oncol. 2014 , 15:700-12. 10.1016/S1470-2045(14)70189-5

25. Golden EB, Demaria S, Schiff PB, et al.: An abscopal response to radiation and ipilimumab in a patient with metastatic non-small cell lung cancer. Cancer Immunol Res. 2013 , 1:365-72. 10.1158/2326-6066 